\title{
LA OCUPACIÓN SOLUTRENSE DEL ABRIGO DE LA BOJA (MULA, MURCIA, ESPAÑA)
}

\section{The Solutrean occupation of La Boja rock shelter (Mula, Murcia, Spain)}

\author{
Armando Lucena ${ }^{1}$, Susana Martínez ${ }^{1}$, Diego E. Angelucci ${ }^{2}$, Ernestina Badal ${ }^{3}$, \\ Valentín Villaverde ${ }^{3}$, Josefina Zapata ${ }^{4}$ y João Zilhão ${ }^{5}$
}

Recibido el 12 de marzo de 2013. Aceptado el 15 de julio de 2013

Resumen. El relleno pleistocénico del abrigo de la Boja (ADB) empieza con un nivel adscrito al Magdaleniense superior, seguido de un potente paquete con ocupación difusa bajo el cual se desarrolla una secuencia depositada durante el último máximo glacial caracterizada por una serie de estructuras de combustión, de tipo hogar plano/amorfo (open hearth), existiendo también hogares de cubeta. Destaca un hogar enlosado, completo y muy bien conservado, excavado en 2012 y adscrito provisionalmente al Solutreogravetiense. Los niveles solutrenses subyacentes son ricos en elementos de adorno, entre los cuales conchas perforadas de Littorina obtusata y Smaragdia viridis; su industria litica incluye raspadores, algunas puntas, y escasos buriles. La datación 14C de muestras de carbón de Juniperus sp. sitúa este paquete entre $16990 \pm 70 B P$ (VERA-5364a), a techo, y $20980 \pm 120$ BP (VERA-5366), a muro.

Palabras clave: hogar, adornos, Solutrense, Solutreogravetiense, radiocarbono.

Abstract. The Pleistocene fill of the La Boja rock shelter (ADB) starts with an Upper Magdalenian level, followed by a thick package with poorly defined occupations under which there is a Last Glacial Maximum sequence with combustion features, mostly of the open hearth type. A particularly well preserved and seemingly complete stone-paved hearth of probable Solutreogravettian age was excavated in 2012. The abundant ornaments in the Solutrean levels include pierced Littorina obtusata and Smaragdia viridis shells and the stone tool assemblages feature endscrapers, some points and rare burins. The $14 \mathrm{C}$ dating of Juniperus sp. charcoal samples places this sequence between $16990 \pm 70$ BP (NERA-5364a), at the top, and $20980 \pm 120$ BP(VERA-5366), at the bottom.

Keywords: hearth, ornaments, Solutrean, Solutreogravettian, radiocarbon.

\section{INTRODUCCIÓN}

En noviembre de 2005, Matías Raja y João Zilhão identificaron en el valle de Rambla Perea una línea de abrigos con materiales paleolíticos en superficie. Los yacimientos se hallan en el contacto entre la base de una escarpa, que corresponde a la pared modelada en calcarenitas del Mioceno superior que delimita el margen izquierdo de la rambla, y la parte superior de una ladera estabilizada del Pleistoceno superior.

(1) Mestrado em Arqueologia. Faculdade de Ciências Humanas e Sociais. Universidade do Algarve. Campus de Gambelas E-8005-139 Faro (Portugal).

(2) Dipartimento di Lettere e Filosofia. Università degli Studi di Trento, via T. Gar 14, I-38122 Trento (Italy).

(3) Departamento de Prehistoria y Arqueologia. Universidad de Valencia. Av. Blasco Ibañez 28. E-46010 Valencia (España).

(4) Área de Antropología Física. Facultad de Biología. Universidad de Murcia. Campus de Espinardo. E-30100 Murcia (España).

(5) Departament de Prehistòria, Història Antiga i Arqueologia. Facultat de Geografia i História. Universitat de Barcelona/ICREA. Carrer Montalegre 6. E-08001 Barcelona (España). 
Desde 2007, en el marco de un proyecto para el estudio de la transición del Paleolítico medio al superior en la Región de Murcia (Zilhão y Villaverde 2008; Zilhão et al. 2010), nuestro equipo ha realizado sondeos y después excavado dos de estos abrigos: Finca de Doña Martina (FDM) y Abrigo de la Boja (ADB). El segundo, objeto de este trabajo, está ubicado unos $50 \mathrm{~m}$ aguas abajo del primero, y sus coordenadas geográficas aproximadas son: $01^{\circ} 29^{\prime} 20^{\prime \prime} \mathrm{W}$ (GDE) y $38^{\circ} 04^{\prime}$ 48" N (Figs. 1 y 2).

\section{DESCRIPCION DE LOS TRABAJOS Y METODOLOGÍA}

La excavación de ADB se inició en 2008 con la abertura en los cuadros S-T/4-5 de un sondeo hasta la cota de unos $2 \mathrm{~m}$ por debajo de la superficie, alcanzada en S5. Debido a la naturaleza del relleno, caracterizado por un sedimento muy suelto, localmente clasto-soportado, hubo que ampliar el área de trabajo, tarea a la que se dedicaron las campañas de 2009 y 2010 . Por cuestiones de seguridad de los perfiles y de los excavadores se adoptó una estrategia de profundización de la excavación en escalones, ya que la textura arenosa suelta del relleno acarreaba peligro de colapso y aconsejaba mantener el espesor máximo de los perfiles por debajo de los $2 \mathrm{~m}$.

Al final de la campaña de 2010 pudimos ampliar la excavación de los niveles pleistocénicos a una parrilla de 3x3 m (S-T-U/4-5-6), lo que permitió plantear el reconocimiento de la estratigrafía en profundidad. Sin embargo, por problemas de financiación, esta tarea no pudo llevarse a cabo hasta el año 2012, cuando pudimos sondear los cuadros T5 y T6 hasta un nivel de bloques conteniendo materiales de tipología auriñaciense y situado a una profundidad de $2 \mathrm{~m}$ por debajo de la cota alcanzada en 2010. Las indicaciones de la geomorfología local apuntan a que todavía no se ha llegado a la base de la secuencia. Si estos bloques corresponden a un horizonte de desplome de la bóveda, podrán existir niveles del Paleolítico medio por debajo de la secuencia reconocida, que abarca ya todo el Paleolítico superior.

Toda la tierra fue cribada en seco con tamiz de malla fina $(2 \mathrm{~mm})$ para la recuperación de restos de talla de pequeño tamaño, de carbones, y de pequeños fragmentos de hueso. De los niveles más intensamente antropizados, ricos en carbones y cenizas, guardamos también el sedimento que pasó por la malla fina, para su flotación con el objetivo de recuperar restos de semillas y frutos carbonizados. Para el registro de materiales en la base de datos se aplicó el protocolo de atribuir entrada individualizada, con procedencia tridimensional, a los hallazgos de las siguientes categorías: útiles retocados, núcleos, hojas enteras o proximales, huesos con más de $5 \mathrm{~cm}$ de largo, objetos de adorno y carbones grandes (para posible datación por radiocarbono; tabla 1). Para un mejor control de las distribuciones espaciales, se subdividieron las unidades estratigráficas en capas artificiales de $5 \mathrm{~cm}$ de espesor (o menor, y variable, cuando era preciso para respetar los límites naturales de la unidad subyacente) y los cuadros en subcuadros de $50 \mathrm{~cm}$ de lado designados en función de un norte convencional definido por el eje de ordenadas.

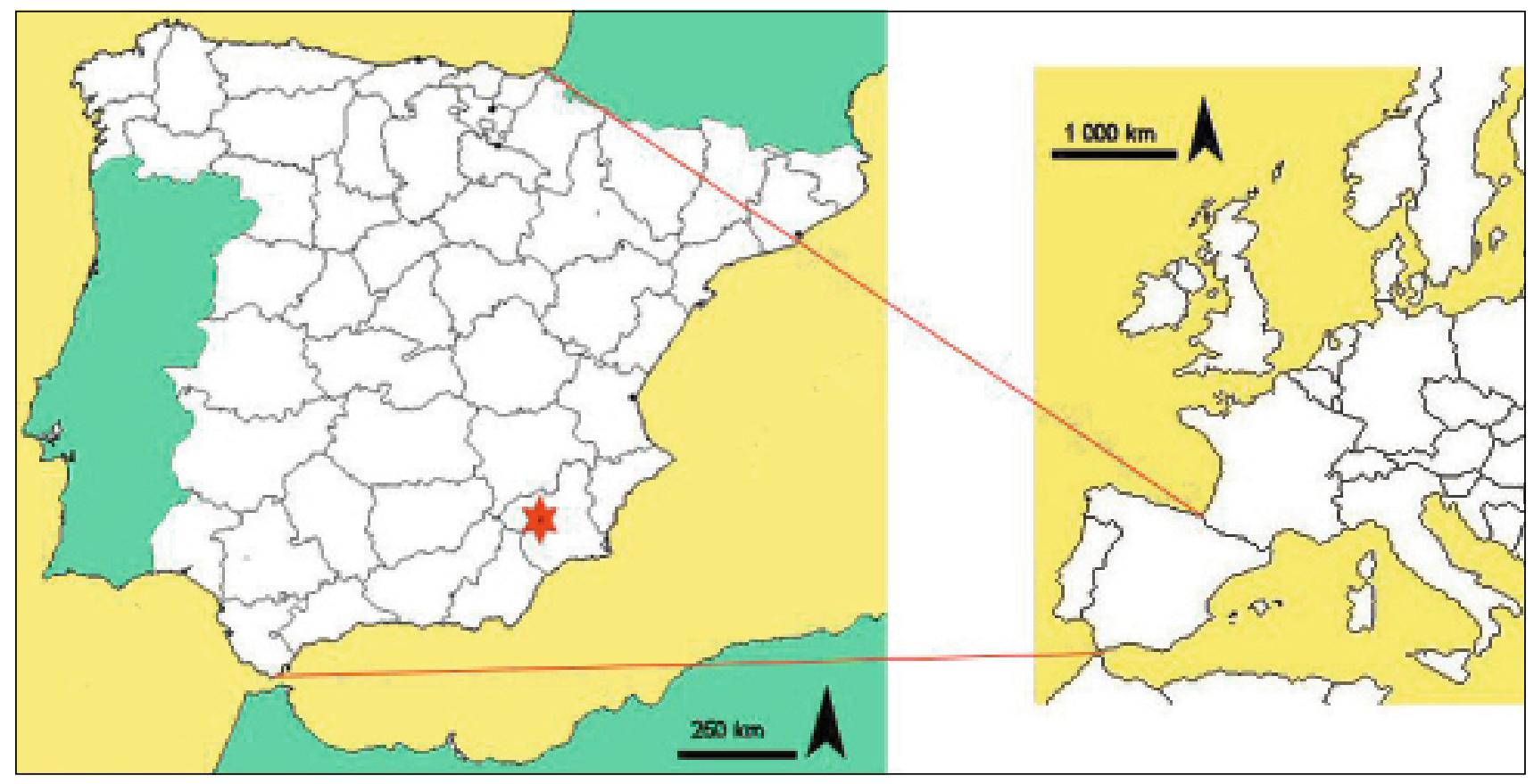

A Figura 1. Ubicación del Abrigo de La Boja en la Península Ibérica. 


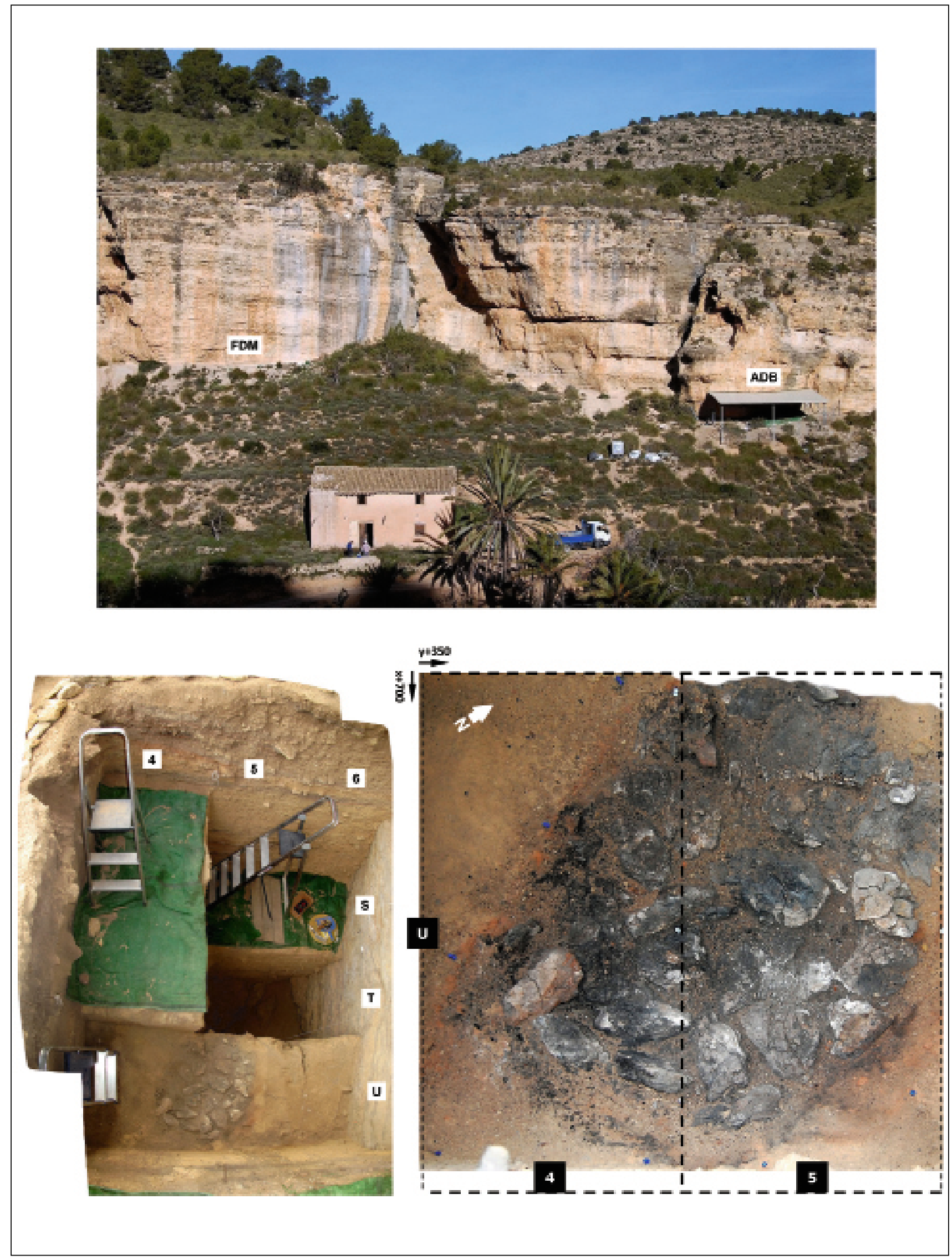

\ FIguRa 2. Arriba: vista sobre los abrigos de Finca Doña Martina y La Boja al final del año de 2012, durante los trabajos de cierre de este último. Abajo, izquierda: vista sobre el área de excavación en La Boja al final de la campaña de septiembre de 2012. Abajo, derecha: planimetría (fotografía ortorectificada) del hogar enlosado en cubeta del nivel SW18B1. 


\begin{tabular}{|c|c|c|c|c|c|c|c|c|c|c|c|c|c|}
\hline Muestra & Nivel & Capa & Cuadro & $\mathrm{x}$ & $Y$ & $\mathrm{Zr}$ & Taxon & Lab \# & Edad BP & $\begin{array}{c}\text { cal BP } \\
(-2 \sigma)\end{array}$ & $\begin{array}{l}\text { cal BP } \\
(+2 \sigma)\end{array}$ & $\delta 13 \mathrm{C}$ & Observaciones \\
\hline $2008-775$ & D & & T5-SE & & & -205 & $\begin{array}{l}\text { Olea } \\
\text { europaea }\end{array}$ & 0xA-20116 & $6959 \pm 33$ & 7694 & 7919 & -23.72 & madriguera \\
\hline \multirow[t]{3}{*}{\begin{tabular}{|l|}
$2010-27$ \\
\end{tabular}} & D & A3 & U5-SE & 75 & 25 & -208 & Juniperus sp. & VERA-5363 & $12605 \pm 45$ & & & $-21,2 \pm 1,1$ & \\
\hline & & & & & & & & VERA-5363_2 & $12585 \pm 40$ & & & $-20,5 \pm 1,1$ & repetición \\
\hline & & & & & & & & VERA-5363_av & $12595 \pm 30$ & 14562 & 15183 & & promedio \\
\hline $2008-774$ & Dbase & & T5-SE & & & -211 & Pinus nigra & VERA-5212a & $12965 \pm 40$ & 15091 & 16169 & $-21,4 \pm 0,7$ & \\
\hline 2012-385 & $E(S W 18 B 1)$ & A11 & U5-SW & 13 & 10 & -273 & Juniperus sp. & VERA-5788 & $16580 \pm 70$ & 19455 & 20027 & $-20,5 \pm 0,9$ & $\begin{array}{l}\text { hogar enlosado en } \\
\text { U/4-5 }\end{array}$ \\
\hline 2010-183 (1) & $\mathrm{E}(\mathrm{SW} 18 \mathrm{~B} 2)$ & A11 & T5-SE & 75 & 10 & -274 & Juniperus sp. & VERA-5364a & $16990 \pm 70$ & 19890 & 20391 & $-19,5 \pm 0,5$ & \\
\hline 2010-183 (2) & & & & & & & Juniperus sp. & VERA-5364b & $17430 \pm 70$ & 20384 & 21203 & $-15,1 \pm 0,7$ & \\
\hline \multirow[t]{3}{*}{$2010-225$} & E(SW18C) & A14 & T5-SW & 3 & 46 & -295 & Juniperus sp. & VERA-5365 & $19390 \pm 100$ & & & $-20,9 \pm 0,6$ & \\
\hline & & & & & & & & VERA-53652 & $19240 \pm 90$ & & & $-19,0 \pm 0,9$ & repetición \\
\hline & & & & & & & & VERA-5365av & $19305 \pm 65$ & 22602 & 23408 & & promedio \\
\hline \multirow[t]{2}{*}{ 2008-760 } & E(SW18E) & A 27 & S5-SE & & & -323 & Juniperus sp. & VERA-5213 & $20980 \pm 110$ & 24564 & 25468 & $-25,4 \pm 0,9$ & \\
\hline & & & & & & & & VERA-5213HS & $21060 \pm 110$ & & & $-22,7 \pm 0,5$ & ácidos húmicos \\
\hline \multirow[t]{4}{*}{\begin{tabular}{|l|}
$2010-316$ \\
\end{tabular}} & E(SW18E) & A19 & T5-SE & 84 & 17 & -316 & Juniperus sp. & VERA-5366 & $20980 \pm 120$ & & & $-21,5 \pm 0,6$ & \\
\hline & & & & & & & & VERA-53662 & $20830 \pm 110$ & & & $-22,0 \pm 0,5$ & repetición \\
\hline & & & & & & & & VERA-5366av & $20900 \pm 80$ & 24513 & 25192 & & promedio \\
\hline & & & & & & & & VERA-5366HS & $20640 \pm 110$ & & & $-20,9 \pm 0,6$ & ácidos húmicos \\
\hline \multirow[t]{2}{*}{ 2012-622 } & E(SW18I) & A36 & T5-NE & 99 & 54 & -384 & Juniperus sp. & VERA-5789 & $27620 \pm 230$ & 31312 & 32495 & $-21,9 \pm 0,8$ & \\
\hline & & & & & & & & VERA-5789HS & $26760 \pm 230$ & & & $-21,8 \pm 0,7$ & ácidos húmicos \\
\hline
\end{tabular}

$\Delta$ Tabla 1. Dataciones 14C para el Abrigo de La Boja. La calibración de los resultados usó el software Calib 6.1.0 (Stuiver y Reimer, 1993) con la curva INTCAL09 (Reimer et al. 2009).

\section{ESTRATIGRAFIA}

En los perfiles sur y oeste, la potencia estratigráfica reconocida mide ya más de $4 \mathrm{~m}$ (Fig. 3). De techo a muro, la secuencia comienza con el revuelto holoceno (unidad A), bajo el cual hay unos niveles coluvionados intermedios (unidades $B$ y $C$ ). El primer nivel in situ es la unidad D, Magdaleniense superior, fechada en $12605 \pm 45$ BP ( 14700 cal BP; VERA5363) y $12965 \pm 40 \mathrm{BP}(\sim 15600 \mathrm{cal} \mathrm{BP} ; \mathrm{VERA}-5212 \mathrm{a})$ sobre muestras de Juniperus sp. y Pinus nigra, respectivamente. Esta unidad D - de la que ya se ha dado noticia anteriormente (Zilhão et al., 2010) - corresponde a un horizonte de importante antropización del depósito (relacionada con esa ocupación y reflejada en la coloración gris o negra de su base) y estaba atravesada por una extensa red de madrigueras. La excavación de esta red tuvo lugar en el Holoceno, como indica la datación de 6959 \pm 33 BP ( $\sim 7800$ cal BP; 0xA20116) obtenida para una muestra de Olea europaea recogida en la base de una cámara del subcuadro SE del cuadro T5.

Por debajo de esta importante ocupación magdaleniense, el relleno pleistocénico ha sido designado como unidad E (unidad geoarqueológica SW18) y es muy homogéneo, estando constituido en su práctica totalidad por productos de la degradación de la pared - arenas limosas amarillas con clastos angulosos y bloques. Su subdivisión estratigráfica sólo se puede hacer sobre la base de los horizontes de antropización relacionados con la ocupación humana, generalmente marcados por el uso del fuego (termoalteraciones, estructuras de combustión, acumulaciones de cenizas). Hemos podido reconocer hasta ahora 19 de estos momentos, separados por paquetes de espesor variable pero, en general, estériles o muy pobres.

Las facies con combustión o termoalteraciones identificadas hasta ahora han sido provisionalmente designadas por una letra, de SW18B hasta SW18P (Fig. 3), y los niveles que las separan por la combinación de las letras designando las termoalteraciones situadas por arriba y por abajo, separadas por una barra (p. ej., SW18B/C). Estos niveles termoalterados presentan casi siempre una micro-estratificación en la que, de techo a muro, se distinguen unos niveles blancos o grises de cenizas, unas acumulaciones lenticulares de carbones (a veces de dimensiones considerables), y una base rubefacta por la precipitación de óxidos de hierro (indicando temperaturas de combustión superiores a $400^{\circ} \mathrm{C}$ ). En general, se trata de hogares planos/amorfos, de tipo open hearth, instalados directamente sobre el suelo, existiendo también hogares de cubeta y destacando un hogar enlosado, completo y muy bien conservado (Perlès 1977; Corchón 1982; Soler et al. 1990). Por su posición estratigráfica, en el nivel SW18B1, y su datación ${ }^{14} \mathrm{C}$ de $16580 \pm 70$ BP ( 19700 cal BP; VERA-5788), adscribimos provisionalmente al Solutreogravetiense este hogar, constituido por una cubeta de $\sim 1 \mathrm{~m}$ de diámetro y $\sim 10 \mathrm{~cm}$ de profundidad cuyo fondo rubefacto se encontraba revestido de lajas, muy agrietadas por la acción del calor (Fig. 2).

La datación de muestras de Juniperus sp. recogidas en los horizontes de acumulación de carbones de los niveles termoalterados ha permitido fechar el nivel SW18C en $19390 \pm 100$ BP ( 23000 cal BP; VERA-5365), es decir, del Solutrense superior. El nivel SW18E, por su parte, está fechado en $20980 \pm 110$ BP y $20980 \pm 120$ BP ( 25000 cal BP; 


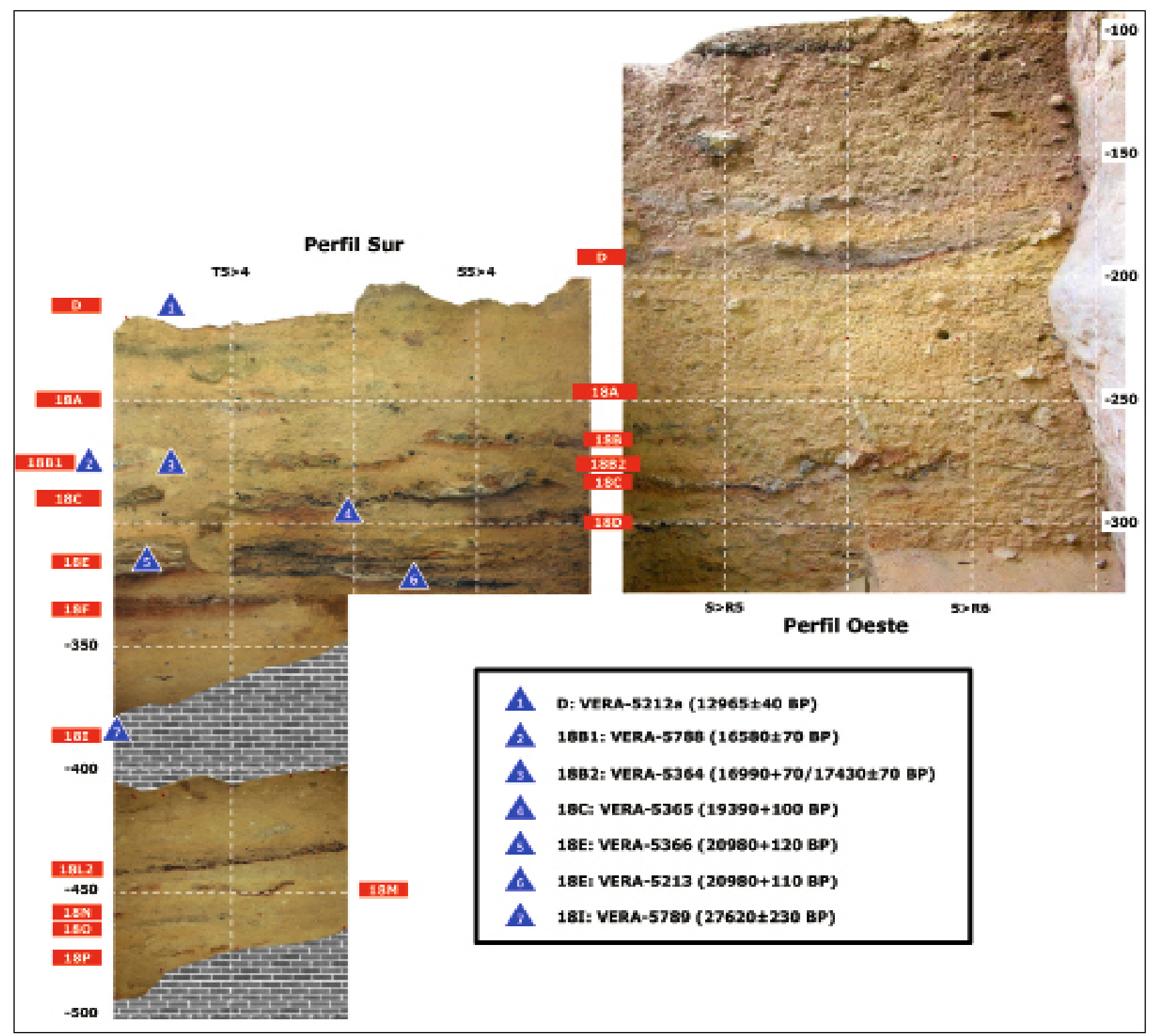

\ Figura 3. Los perfiles estratigráficos oeste y sur de La Boja al final de la campaña de septiembre de 2012 (mosaicos fotográficos ortorectificados; cotas en cm por debajo del nivel cero de excavación). Se indican los niveles de termoalteración y la procedencia de las muestras de estos cuadros que fueron fechadas por el radiocarbono.

VERA-5213 y VERA-5366), es decir, del Solutrense inferior; por su contenido industrial, el nivel SW18E/F es también de esta cronología. Inmediatamente por debajo, en los niveles SW18F, SW18F/G y SW18G, los materiales líticos recogidos (buriles sobre truncadura retocada) son ya de una tipología cuyas características apuntan al Gravetiense; por su posición estratigráfica, unos $40 \mathrm{~cm}$ por encima de SW18I, que está fechado en $27620 \pm 230$ BP ( 31900 cal BP; VERA-5789), estos niveles deben pertenecer a una fase final del tecnocomplejo.

\section{MATERIALES}

En general, los diferentes niveles de ocupación de la unidad E se caracterizan por una cantidad escasa de restos de industria lítica, en lo que contrastan marcadamente con la unidad D, cuyo recuento tipológico, a finales de la campaña de 2009, excavados unos $6 \mathrm{~m}^{2}$, presentaba un total de 122 útiles retocados (Zilhão et al. 2010: tabla 2). En cada uno de los diferentes niveles solutrenses el total de útiles retocados es como mucho de un 10\% de este valor, a pesar de que su área excavada es de unos $4 \mathrm{~m}^{2}$, es decir, inferior en apenas una tercera parte. Esta diferencia puede estar relacionada con un cambio en la naturaleza funcional de las ocupaciones y/o con el hecho de que los sucesivos desplomes de la visera hayan llevado a la progresiva migración hacia la pared de las áreas de actividad. Por ejemplo, la zanja actualmente abierta nos podría haber permitido muestrear el centro de la ocupación del Magdaleniense superior pero no así el centro de las ocupaciones del Solutrense, que posiblemente se extienden 
hacia el exterior y de las que podríamos haber muestreado, hasta el momento, sólo una banda periférica interior.

La industria lítica de los niveles solutrenses es casi exclusivamente en sílex, con raros ejemplos de aprovechamiento de otras rocas (cuarcita, caliza, cristal de roca, calcedonia). Los raspadores son relativamente frecuentes, y los buriles extremadamente raros. Se han hallado algunas puntas, destacando un fragmento con retoque plano cubriente, posiblemente una base de pequeña hoja de laurel, en SW18C, y una típica punta de cara plana en la base de SW18E/F (Fig. 4); de las unidades A (revuelto holocénico) y D (Magdaleniense superior) proceden dos puntas de Parpalló, un fragmento de pieza con retoque bifacial (posiblemente un esbozo de punta de Parpalló rota en proceso de fabricación) y un fragmento basal de micropunta escotada de dorso (Zilhão et al. 2010: fig. 8) en posición secundaria.

En los niveles SW18B, SW18B1, SW18B2, no se encontró material lítico diagnóstico. Sin embargo, la continuidad en las materias primas (especialmente en lo que concierne la preferencia por la calcedonia) y en la tecnología de base, así como la presencia de piezas apuntadas hechas sobre lámina (Fig. 4), sugieren que estamos todavía en la tradición solutrense; nuestra hipótesis es, por lo tanto, que estos niveles pertenecen al Solutreogravetiense y no al Magdaleniense antiguo, como también podría plantearse. Las dataciones absolutas obtenidas son acordes con esta hipótesis, puesto que sitúan estos niveles entre $17430 \pm 70 \mathrm{BP}$ y $16580 \pm 70 \mathrm{BP}$ (Tabla 1), es decir, en fechas idénticas a las indicadas por la datación del hogar solutreogravettiense de la Ratlla del Bubo (Crevillent, Alicante) - $17360 \pm 180$ BP (Ly-5219; Soler et al. 1990) - y anteriores a la más antigua actualmente disponible para el Magdaleniense de la secuencia de referencia de Cendres- $16030 \pm 60$ BP (Beta-287541), para su nivel XII (Villaverde et al. 2012). Sin embargo, no podemos descartar que el intervalo de un milenio por el que se distribuyen los resultados de La Boja esté reflejando un fenómeno de palimpsesto (por hiato en la secuencia, ya sea erosivo o de sedimentación), y que en los niveles SW18B, SW18B1 y SW18B2 se encuentren subsumidas tanto la última ocupación solutreogravettiense como la primera ocupación magdaleniense del abrigo.

Los niveles SW18D y SW18E tampoco han dado fósiles indicadores. De SW18C/D procede un fragmento de pieza microlítica de dorso con posible fractura de impacto (Fig. 4), por lo que el nivel SW18D podría ser también del Solutrense superior, aunque, por estratigrafia, no podamos descartar que pertenezca ya a un Solutrense medio. A pesar de su pobreza industrial en el área excavada, este nivel ha dado abundantes objetos de adorno sobre concha marina y también un fragmento de coral rojo, indicando la existencia de contactos con la costa, ya sea a través de redes de intercambio o de la existencia de un sistema de poblamiento incluyendo territorios litorales y territorios de interior, posiblemente explotados de forma estacional; destaca la presencia de la especie Smaragdia viridis, cuya utilización como adorno era hasta ahora desconocida (o no reconocida?) en el Paleolítico superior ibérico (Fig. 4). La atribución de SW18E al Solutrense inferior resulta de su posición en la secuencia y de su datación absoluta, y es acorde a las características de la industria lítica - faltan piezas de retoque plano cubriente bifacial y la única pieza apuntada es un fragmento mesial con retoques bilaterales cortos que, con toda probabilidad, corresponde a un fragmento de punta de cara plana (Fig. 4).

\section{DISCUSIÓN Y CONCLUSIONES}

En combinación con la serie de dataciones ya obtenida, de total coherencia estratigráfica, el material lítico diagnóstico recuperado indica que en La Boja está representada la secuencia prácticamente completa del Paleolítico superior regional. El único hiato que hasta el momento hemos podido identificar es el indicado por el contacto directo entre el Solutrense inferior de SW18E/F y el Gravetiense de SW18F, SW18F/G y SW18G. A partir de las fechas obtenidas para SW18E y SW18I podemos estimar en unos $12,5 \mathrm{~cm} / \mathrm{mile-}$ nio la tasa de acumulación de la secuencia gravetiense, lo que daría para estos niveles una estimación cronológica de $\sim 23000$ BP en años de radiocarbono (o sea, 28000 cal BP).

Aceptándose este razonamiento, faltarian (por erosión o no sedimentación) unos dos milenios en la secuencia, lo que es acorde con la ausencia de niveles atribuibles a los momentos intermedios de la transición entre Gravetiense y Solutrense (el mal Ilamado "Auriñaciense V" y el Protosolutrense), bien conocidos en secuencias del suroeste de Francia y centro de Portugal, regiones en las que se han podido datar justamente de ese intervalo 23000-21000 BP no representado en La Boja (Zilhão 1997). La ausencia de los fósiles directores de esos momentos (raspadores carenados y laminitas de retoque marginal para el primero, puntas de Vale Comprido para el segundo) en SW18E, SW18E/F, SW18F, SW18F/G y SW18G corrobora esta conclusión.

Una interpretación alternativa, acorde con ciertos modelos cronoestratigráficos que se han propuesto para el desarrollo del Paleolítico superior del Mediterráneo español (Cacho et al. 1980), sería la de una transición directa del Gravetiense al Solutrense, sin aquellos momentos intermedios que se documentan en el mundo atlántico. Eso explicaría lo que se ha interpretado como la perduración de importantes elementos gravetienses (buriles y laminitas de borde abatido) en el Solutrense inferior y medio de Andalucía y Murcia. Lo observado en ADB indica más bien que esa "perduración" refleja unos niveles en los que, por falta de resolución de las secuencias, ocupaciones como las representadas en ADB por los niveles comprendidos entre SW18E y SW18G están subsumidas en palimpsestos formados a lo largo de 


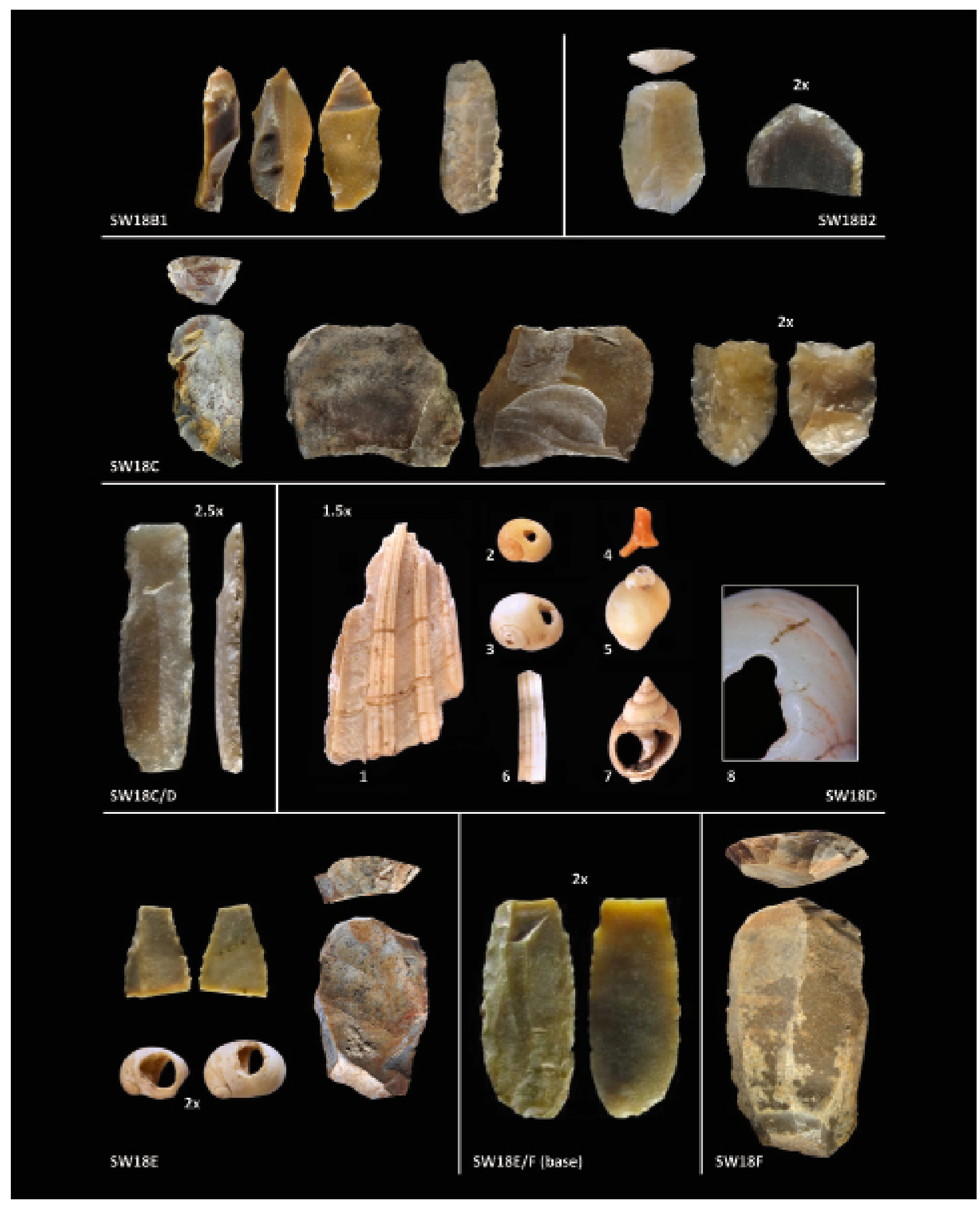

\ Figura 4. Materiales de la secuencia solutrense de La Boja (reproducidos a escala 1:1, excepto cuando indicado). SW18B1: hoja apuntada con fractura de impacto y hoja no retocada de calcedonia. SW18B2: raspadores. SW18C: raspador, pieza esquirlada y base de pieza foliácea con retoque dorsal plano, cubriente (fragmento de pequeña hoja de laurel?). SW18C/D: fragmento de pieza (punta?) microlítica de dorso con fractura de impacto. SW18D: adornos (1. Pecten jacobaeus, fragmento de valva derecha; 2-3. Littorina obtusata perforadas; 4. fragmento de Corallium rubrum (?); 5. concha no perforada; 6. Tubo de Dentalium sp.; 7. Nassarius (Sphaeronassa) mutabilis perforada; 8. detalle de la perforación de una Littorina obtusata, observándose la muesca de desgaste producida por el uso como colgante. SW18E: fragmento de hoja apuntada (punta de cara plana?) y conchas perforadas de Smaragdia viridis. SW18E/F: punta de cara plana con fractura de impacto distal. SW18F: raspador. 
un periodo de tiempo importante. Que, en $A D B$, hayamos podido darnos cuenta del problema se debe a la asociación de los artefactos con niveles de termoalteración muy bien conservados, lo que posibilita una delimitación centimétrica de las ocupaciones. Esto es lo que permitió comprender que SW18F y SW18F/G corresponden a una ocupación distinta de la de SW18E y SW18E/F, aunque estén inmediatamente por debajo, en contacto directo, sin nivel estéril intermedio, al contrario de lo que sucede hacia arriba y hacia abajo. En otras circunstancias, la diferenciación estratigráfica de los dos momentos de ocupación habría sido imposible.

A pesar de esta laguna, y de la muy mala (en los niveles de cenizas) o nula (en los restantes) conservación del hueso, la elevada resolución estratigráfica de la secuencia abre la posibilidad de aclarar dos de las asignaturas pendientes de la crono-estratigrafía del Paleolítico superior mediterráneo: la del orden de aparición de los diferentes fósiles directores del Solutrense y del Solutreogravetiense; y la del timing de la transición entre este último y el Magdaleniense antiguo. Además, la abundancia y excelente conservación de carbones y fitolitos, asi como de la estructuración espacial del hábitat, abre la posibilidad de estudiar los diferentes momentos de ocupación desde un punto de vista paleoetnográfico. Esto supone la ampliación de los trabajos hacia la pendiente, fuera de la protección otorgada por la actual visera, lo que, con el cierre del yacimiento al final de la campaña de 2012
(Fig. 2), será posible en futuras campañas, una vez concluida la fase de reconocimiento de la potencia estratigráfica del abrigo actualmente en curso.

\section{AGRADECIMIENTOS}

Los trabajos que venimos desarrollando en el Abrigo de la Boja han podido realizarse gracias al respaldo financiero y logístico de varias instituciones y a la colaboración de un pequeño pero muy dedicado equipo de excavación. Cabe destacar el apoyo del proyecto HAR2011-24878, del Ministerio de Economía y Competitividad, de las Universidades de Murcia, Valencia, Barcelona y Colonia (Alemania), del Museo de Arte Ibérico El Cigarralejo de Mula, que puso sus instalaciones de laboratorios y alojamiento a disposición del equipo, del Ilmo. Ayuntamiento de Mula y de la Fundación Séneca. Además de los signatarios, los trabajos de excavación contaron con la participación de estudiantes de licenciatura y de posgrado de las Universidades de Murcia, Trento (Italia), Algarve (Portugal) y Bristol (Reino Unido): Maria Mântua, Daniela Anesin, Haidé Martins, Alessandro Poti, Luca Baruffaldi, Marta Tebar y Lorenzo Puche. Para la determinación taxonómica de los adornos sobre concha hemos podido contar con la preciada ayuda de Iván Mulero, de la Universidad de Murcia. •

\section{BIBLIOGRAFÍA}

Villaverde, V., Román, D., Pérez Ripoll, M., Bergadè, M. M. y Real, C. 2012: "The End of the Upper Palaeolithic in the Mediterranean Basin of the Iberian Peninsula". Quaternary International 272-273: 17-32.

CACHO, C. 1980: "Secuencia cultural del Paleolitico Superior en el Sureste español". Trabajos de Prehistoria 37: 65-108.

CORCHÓN, M. S. 1982: "Estructuras de combustión en el Paleolítico: A propósito de un hogar de doble cubeta de la Cueva de Las Caldas (Oviedo)". Zephyrvs XXXIV-XXXV: 27-46.

Perles, C. 1977: Préhistoire du feu. Masson, Paris.

Reimer, P. J., Baillie, M. G. L., Bard, E., Bayliss, A., Beck, J. W., Bertrand, C., Blackwell, P. G., Buck, C. E., Burr, G., Cutler, K. B., Damon, P. E., Edwards, R. L., Fairbanks, R. G., Friedrich, M., Guilderson, T. P., Hughen, K. A., Kromer, B., Mccormac, F. G., Manning, S., Bronk Ramsey, C., Relmer, R. W., Remmele, S., Southon, J. R., Stuiver, M., Talamo, S., Taylor, F. W., Van Der Plicht, J. y Weyhenmeyer, C. E. 2009: "Intcal09 and Marine09 Radiocarbon Age Calibration Curves, 0-50,000 Years Cal BP". Radiocarbon 51 (4): 1111-1150.
SOler, B., Badal, E., Villaverde, V. y Aura, E. 1990: "Nota sobre un hogar solútreo-gravetiense de la Ratlla del Bubo (Crevillent, Alicante)". Archivo de Prehistoria Levantina 20: 79-93.

Stuiver, M. y Reimer, P. J. 1993: "Extended ${ }^{14} \mathrm{C}$ Data Base and Revised CALIB 3.0 ${ }^{14} \mathrm{C}$ Age Calibration Program. Radiocarbon, 35(1): 215-230.

Villaverde, V., Román, D., Pérez Ripoll, M., Bergada, M.M. y Real, C. 2012. "The End of the Upper Palaeolithic in the Mediterranean Basin of the Iberian Peninsula". Quaternary International 272-273: 17-32.

ZııнÃo, J. 1997: O Paleolítico Superior da Estremadura portuguesa. Colibri, Lisboa.

Zilhão, J. y VillaVerde, V. 2008: "The Middle Paleolithic of Murcia". Treballs d'Arqueologia 14: 229-248.

Zilhão, J., Angeluccl, D., Badal, E., Lucena, A., Martín, I., Martinez, S., VILLAVERDE, V. y ZAPATA, J. 2010: "Dos abrigos del Paleolítico superior en Rambla Perea (Mula, Murcia)". En X. Mangado (ed.) El Paleolítico superior peninsular. Novedades del siglo XXI. Universidad de Barcelona, Barcelona: 137-148. 